\title{
Correlative TEM and APT of Helium Bubbles in Ion-Irradiated RAFM Steel
}

\author{
B. Mazumder ${ }^{1}$, C. M. Parish ${ }^{2}$ and M. K. Miller $^{1}$ \\ ${ }^{1 .}$ Center for Nanophase Materials Sciences, Oak Ridge National Laboratory, Oak Ridge, TN, USA. \\ 2. Materials Science and Technology Division, Oak Ridge National Laboratory, Oak Ridge, TN, USA.
}

Neutron irradiation of structural materials introduces He through $(n, \alpha)$ transmutation, which degrades the mechanical properties through swelling, blistering, and He embrittlement [1]. He bubbles are trapped on grain boundaries and dislocations, degrading properties; recent studies show that trapping on the surfaces of nanoclusters and larger precipitates [2] may mitigate materials degradation. Hence, it is important to investigate He bubble distributions in materials used in current reactors, such as reduced activated ferritic-martensitic (RAFM) steels. (S)TEM and APT are powerful tools to study microstructural features and provide complementary information. Correlative APT-TEM studies [3] have illustrated the direct comparison of the same features visible via each technique, which can provide more reliable spatial and chemical information. Here, preliminary results correlating TEM and APT of helium bubbles are shown.

The material used in this study was F82H steel, He-irradiated to $~ 8000$ ppm He, $\sim 0.5$ dpa at $500{ }^{\circ} \mathrm{C}$ [4]. (S)TEM images of F82H before and after $\mathrm{He}$ ion irradiation are presented in Fig. 1a and 1b, respectively. Bubbles or voids, with a size range of $4-16 \mathrm{~nm}$, are visible (circular features). APT at the same depth shows the presence of bubbles, some with diameters $<2 \mathrm{~nm}$ [4]. Since the specimens were prepared from different locations, direct correlation is impossible. For direct APT-(S)TEM correlation, a needle-shaped specimen at a depth of $500 \mathrm{~nm}$ from the surface was prepared, and both TEM and APT were performed (TEM, Fig. 2a). Within this volume, bubbles with diameters ranging from 2-8 $\mathrm{nm}$ (3.8 $\mathrm{nm} \pm 0.8 \mathrm{~nm}$ average) were measured via TEM; those with diameters $<2 \mathrm{~nm}$ were not detected. Subsequently, APT analysis was performed on the same specimen, and Fig. 2b shows the He bubble distribution from the analyzed volume shown in Fig 2a. Bubble sizes in the APT-analyzed volume ranged from $1 \pm 0.5-7 \pm 1.2 \mathrm{~nm}$, with an effective average bubble diameter of $3.5 \pm 0.4 \mathrm{~nm}$. APT data revealed a bubble number density of $7.2 \times 10^{22} \mathrm{~m}^{-3}$, which is roughly twice that measured by TEM, $3 \times 10^{22} \mathrm{~m}^{-3}$. This discrepancy is likely because imprecision in determining the TEM-analyzed volume. The size variation of the bubbles measuredby both the techniques are compared in Fig 2c and 2d, and exhibit similar size distributions for large $(>2 \mathrm{~nm})$ bubbles, but APT detected comparatively more bubbles in the smaller 1-2 nm size range. This correlative study provides more precise information on He bubble locations and distributions within the matrix and other microstructures (precipitate, grain boundary, etc.) if present within the analysis volume.

Future microstructural engineering for He mitigation will require that the locations of the He bubbles be determined and correlated using both techniques. He bubble features analyzed in (S)TEM (e.g., dislocations or loops, grain boundaries, chemically similar but crystallographically distinct precipitates) and features analyzed in APT (e.g., boundaries with different Gibbsian segregation, extremely small precipitates) can be successfully correlated, opening up new paths for strategy developments in designing of He-tolerant microstructures. This example illustrates a first step toward that goal. Future TEM or STEM tomography experiments will provide more quantitative comparisons. 
References:

[1] GR Odette et al, Annual Review of Material Research 38 (2008) p. 471.

[2] Q Li et al, Journal of Nuclear Materials 445 (2014) p. 165, and references therein.

[3] I Arslan et al, Ultramicroscopy 108 (2008) p. 1579.

[4] B Mazumder et al, Nuclear Materials and Energy 1 (2015) p. 8.

[5] Research supported by the U.S. Department of Energy, Office of Basic Energy Sciences, Materials Sciences and Engineering Division. APT and TEM conducted as part of a user proposal at ORNL's Center for Nanophase Materials Sciences (CNMS), which is a DOE Office of Science User Facility.
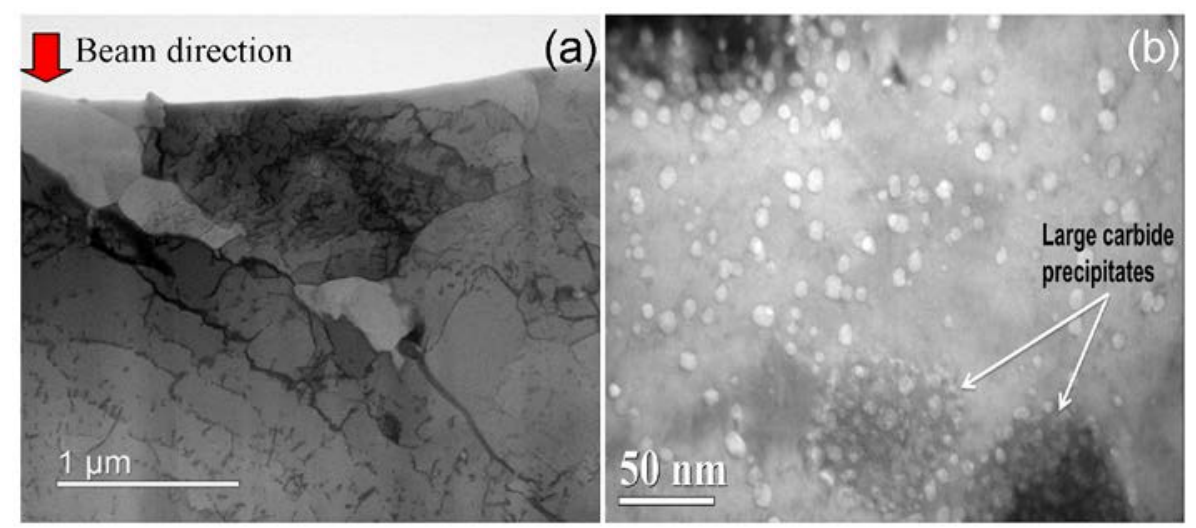

Figure 1. (a) BF-STEM image of F82H steel before He ion irradiation, b) under-focused TEM image of F82H after He ion irradiation revealing the distribution of He bubbles in the matrix and carbides.

(a)
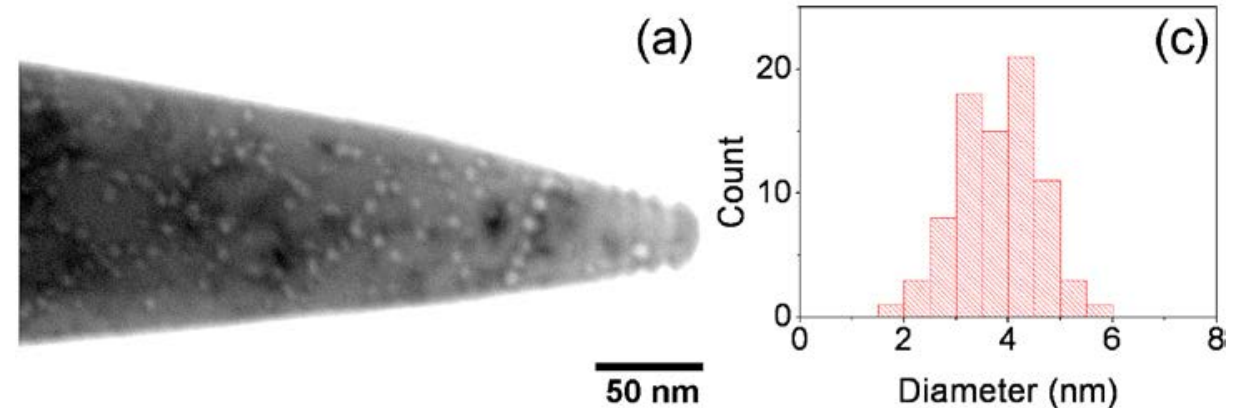

(b)
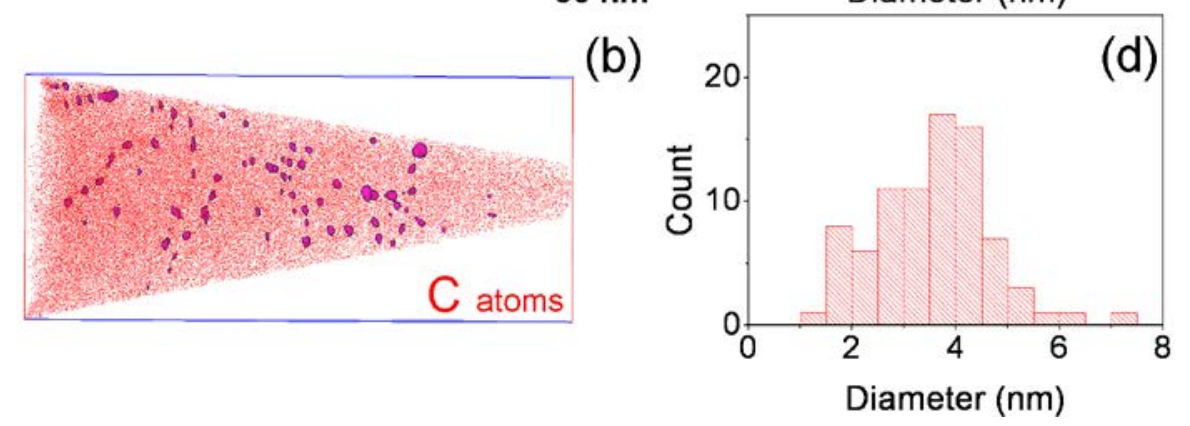

Figure 2. (a) TEM image in the under-focused condition showing He bubbles in the APT needle-shaped specimen prior to APT analysis. (b) APT reconstruction of the same needle. Density isosurfaces (purple) at $65-70$ atoms/nm reveal He bubbles. In TEM, the entire specimen can be imaged, whereas in APT a partial volume is analyzed due to the local electrode and the detector size. Size distribution of the detected He bubbles within the volume analyzed by (c) TEM and (d) APT. 